\title{
In Situ Observation and Measurement of Actin Stress Fiber Deformation in Stretched Osteoblast like Cell
}

\author{
Katsuya Sato ${ }^{1}$, Kenta Nunobiki², Shoichiro Fujisawa ${ }^{1}$, Tasuku Nakahara ${ }^{3}$, Kazuyuki Minami ${ }^{3}$ \\ ${ }^{1}$ Graduate School of Technology, Industrial and Social Sciences, Tokushima University, Tokushima, Japan \\ ${ }^{2}$ Graduate School of Advanced Technology and Science, Tokushima University, Tokushima, Japan \\ ${ }^{3}$ Graduate School of Sciences and Technology for Innovation, Yamaguchi University, Ube, Japan \\ Email:katsuyas@tokushima-u.ac.jp
}

How to cite this paper: Sato, K., Nunobiki, K., Fujisawa, S., Nakahara, T. and Minami, K. (2017) In Situ Observation and Measurement of Actin Stress Fiber Deformation in Stretched Osteoblast like Cell. Advances in Bioscience and Biotechnology, 8, 421-433. https://doi.org/10.4236/abb.2017.811031

Received: October 3, 2017

Accepted: November 18, 2017

Published: November 21, 2017

Copyright $\odot 2017$ by authors and Scientific Research Publishing Inc. This work is licensed under the Creative Commons Attribution International License (CC BY 4.0).

http://creativecommons.org/licenses/by/4.0/

\begin{abstract}
It is believed that mechanical stimuli, such as stretching of the extracellular matrix, are transmitted into cells via focal adhesion complexes and the actin cytoskeleton. Transmission dynamics of strain from the extracellular matrix into intracellular organelles is crucial to clarify the mechanosensing mechanisms of cells. In this study, we observed deformation behavior of actin stress fibers under uniaxial stretch using an originally developed cell-stretching microelectromechanical system (MEMS) device. It was difficult to conduct in situ observation of cells under stretch using conventional cell stretching devices, because motion artifacts such as rigid displacement during stretch application were not negligible. Our novel cell-stretching MEMS device suppressed rigid displacement while stretching, and we succeeded in obtaining time-lapse images of stretched cells. Uniaxial strain with a $10 \%$ magnitude and strain rate of $0.5 \% / \mathrm{sec}$ was applied to cells. Deformation behaviors of the cells and actin stress fibers were recorded using a confocal laser scanning microscope. In time-lapse images of stretched cells, strains along each stress fiber were measured manually. As a result, in cells with a relatively homogeneous stress fiber structure oriented in one direction, distribution of the axial strain on stress fibers generally corresponded to deformation of the stretching sheet on which the cells had adhered. However, in cells with a heterogeneous stress fiber structure oriented in several directions, we found that the strain distribution along stress fibers was not homogeneous. In regions around the cell nucleus, there was a more complicated strain distribution compared with other regions. Our results suggest the cell nucleus with a stiff mechanical resistance yields such a complicated strain distribution in stress fibers.
\end{abstract}




\section{Keywords}

Actin Cytoskeleton, Stretching Stimuli, Osteoblast, Cell Biomechanics, MEMS

\section{Introduction}

The actin cytoskeleton plays important roles in various cellular features such as motility and morphology [1] [2], and its dynamic reorganization is crucial for their activities. Stress fibers, a kind of actin cytoskeletal fiber, not only perform these roles, but also act as an important signaling pathway in mechanotransduction through which cells sense mechanical stimuli such as force and deformation [3]. It has been suggested that actin stress fibers transmit force or deformation from the extracellular environment into intracellular organelles [4]. Therefore, clarifying the transmission characteristics of extracellular force or deformation into intracellular organelles via actin stress fibers is very important to further understand mechanotransduction mechanisms in cells.

Changes in the surrounding mechanical environment are known to induce actin stress fiber reorganization. For example, the alignment direction of actin stress fibers reorganizes dynamically under cyclic stretch application or shearing fluid flow [5] [6]. Understanding the intracellular distribution of deformation or force in actin stress fibers under mechanical perturbation is crucial to reveal the relationship between dynamic actin remodeling, including highly localized polymerization/depolymerization of actin molecules, and intracellular mechanical conditions.

In conventional experimental setups, stretching stimulus causes non-negligible motion artifacts during stretch application, such as loss of focus or moving out of the field of view [7]. These motion artifacts disturb continuously in situ time-lapse observations of actin stress fiber dynamics under stretch application with high spatial and temporal resolution. In the present study, we developed a novel cell-stretching microelectromechanical system (MEMS) device to reduce motion artifacts during stretch application and succeeded in conducting precise time-lapse imaging of actin stress fiber dynamics under uniaxial stretching [8]. The distribution of axial strain in stress fibers was manually calculated from precise time-lapse image data. In addition, we examined the difference between the actual strain distribution in actin stress fibers and the estimated strain distribution calculated from deformation of the extracellular stretching sheet.

\section{Materials and Methods}

\subsection{Cell Culture}

MC3T3-E1 osteoblast-like cells (provided by RIKEN BRC through the National Bio-Resource Project of the Ministry of Education, Culture, Sports, Science and Technology) were cultured in $\alpha$-modified Eagle's medium ( $\alpha$-MEM; Nacalai Tesque) 
supplemented with $10 \%$ fetal bovine serum. For subculture, cells were cultured in a $25 \mathrm{~cm}^{2}$ culture flask at $5 \% \mathrm{CO}_{2}$ with $37^{\circ} \mathrm{C}$ and $100 \%$ humidity. Prior to the experiment, cells were seeded onto a fibronectin-coated $35 \mathrm{~mm}$ glass-bottom dish, on which our originally developed cell stretching MEMS devices had been fabricated, at $1 \times 10^{5}$ cells/dish. After 18 hours of preincubation for sufficient cell adhesion, the Lifeact-TagGFP2 fusion protein plasmid DNA (Ibidi) was transfected by following the procedure described below.

\subsection{Transfection Procedure to Visualize the Actin Cytoskeleton}

Lifeact-TagGFP2 plasmid DNA was transiently transfected into cells to visualize the actin cytoskeleton by lipofection using FuGENE HD transfection Regent (Promega). Cells were incubated in $\alpha$-MEM containing $0.5 \mu$ p plasmid DNA and $2 \mu \mathrm{l}$ FuGENE HD transfection regent for 18 hours. Prior to the experiment, transfection medium was replaced with normal culture medium.

\subsection{Cell-Stretching MEMS Device and Stretch Condition}

The cell-stretching MEMS device was slightly modified from our previously reported device [8]. Figure 1 shows a schematic illustration of the device. Six devices were fabricated onto a $22 \mathrm{~mm}$ square coverslip glass that was glued to a 35 $\mathrm{mm}$ dish with a $\phi 18 \mathrm{~mm}$ hole at the bottom. As shown in Figure 1(b), each microdevice consisted of one pair of arms fabricated from photoresist SU-8 and a cell-stretching transparent silicone elastomer sheet of approximately $5 \mu \mathrm{m}$ in thickness. Figure 2 shows a microscopic image of the cell-stretching MEMS device and the tips of two metal needles. The tips of the metal needles were set on the device arms. The needle on the right side was held by the micromanipulator mounted on the microscope stage to fix one end of the silicone stretching sheet. The needle on the left side was held by the micromanipulator via a piezo electric actuator (MC-140L; Mess-tek) to apply uniaxial stretch to the silicone sheet with

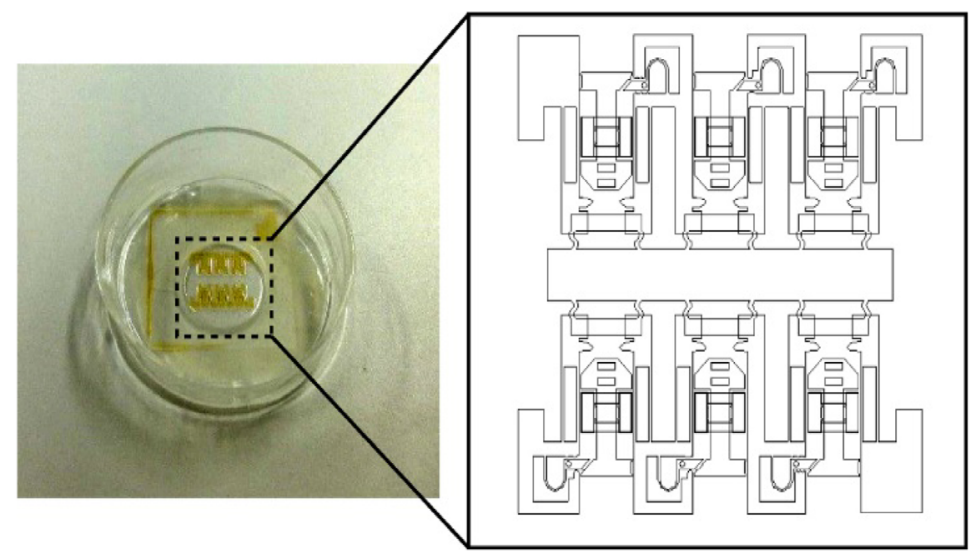

(a)

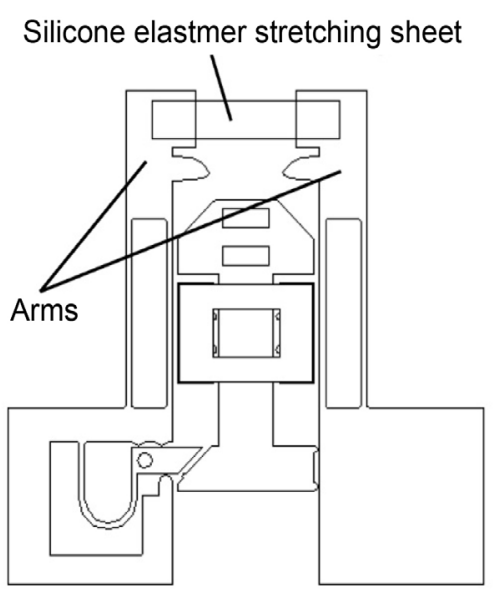

(b)

Figure 1. Schematics of the cell-stretching MEMS devices on a coverslip attached to a $35 \mathrm{~mm}$ dish. (a) Arrangement of six cell-stretching MEMS devices; (b) Magnified schematic of the device. 


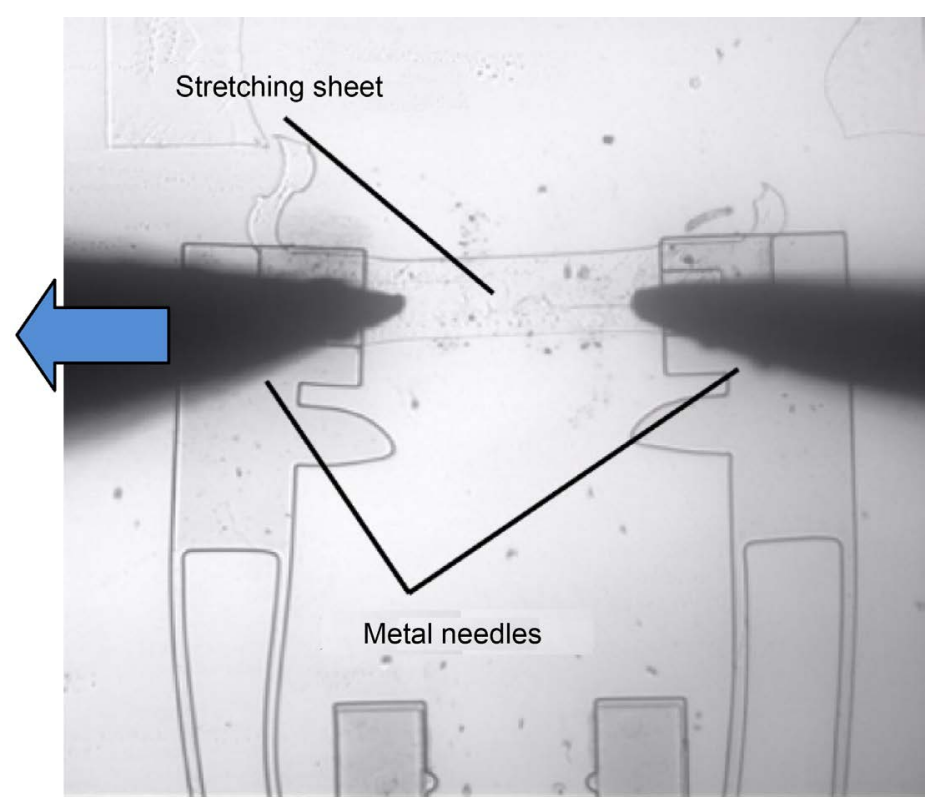

Figure 2. Stretching setup of metal needles and stretch device. The needle on right side was fixed and the needle on left side is slid to the left direction to apply stretch to the stretching sheet by using piezo electric actuator.

a controlled strain magnitude and rate. The target strain magnitude was set to $10 \%$ and the strain rate was a constant $0.5 \% /$ second. Although we precisely controlled displacement of the metal needle to apply stretch to the stretching sheet using the PZT actuator, the actual strain magnitude on the stretching sheet did not match the target magnitude because of backlash between the needle tip and the device arm. Therefore, we measured the actual nominal strain magnitude on the stretching sheet in time-lapse images of the transmitted image in which we could observe deformation behavior of the stretching sheet.

\subsection{Image Acquisition and Analysis}

Time-lapse fluorescence images of the actin cytoskeleton were obtained using an inverted confocal laser scanning microscope (A1R; Nikon) with a $\times 60$ oil immersion objective lens. Lifeact-TagGFP2 was excited by a $488 \mathrm{~nm}$ Ar Laser, and fluorescence was detected through a $525 \mathrm{~nm}$ band-pass filter with a $50 \mathrm{~nm}$ band width. Two images of Lifeact-TagGFP2 and a transmitted image were acquired simultaneously using two independent photomultiplier tubes. The confocal pinhole of the photomultiplier was fully open, and images were obtained by quasi-epifluorescence microscopy (estimated thickness of the optical section was $2.32 \mu \mathrm{m}$ ). The recorded image size was $1024 \times 256$ pixels with 12 bit resolution. The image acquisition rate was approximately 1.88 frames/second. All experiments were conducted in a normal atmosphere at room temperature (approximately $24^{\circ} \mathrm{C}$ ). To avoid any influences on the cell conditions, image acquisition was completed within half an hour.

Obtained time-lapse images were analyzed using ImageJ software (NIH). De- 
formation behavior of stress fibers was evaluated by axial strain along a fiber and the fiber angle between stretching and fiber axes. The axial strain was calculated from the change in distance between two tracing markers on the target stress fiber using the manual measure function in ImageJ. Stress fibers with noticeable branches or nodes were selected as the strain measured fibers because these branches and nodes could be used as clear tracing markers. The distance between two tracing markers was considered as the representative length of the target stress fiber. Axial strain $\varepsilon_{F}$ was defined by $\left(L_{2}-L_{1}\right) / L_{1}, L_{1}$ is the initial length of the fiber, and $L_{2}$ is the length of the fiber after stretching. Nominal strain on the silicone stretching sheet $\varepsilon_{s}$ was measured in the transmitted image that showed a contrast image of the cell and silicone sheet together.

\subsection{Estimation of Displacement Distribution on the Stretching Sheet}

To compare the difference between deformation behaviors of actin stress fibers and the silicone stretching sheet during uniaxial stretch application, we estimated the displacement distribution on the stretching sheet. Four noticeable spot marks were selected on the sheet, which were located around the target cell, to form a rectangular shape. Displacement of these spot marks was traced during stretch application. We hypothesized that displacement was homogeneously distributed in the enclosed rectangle. Displacement of an arbitrary point could be estimated by deformation of the rectangle.

\section{Results}

\subsection{Deformation Dynamics of Actin Stress Fibers in a Stretched Cell}

Representative time-lapse fluorescence images of actin stress fibers in a uniaxially stretched osteoblast-like cell are shown in Figure 3. X- and y-axes were defined as indicated in the figure. Stretch was applied in the $\mathrm{x}$-axis direction. The strain magnitude $\mathcal{E}_{s}$ shows the nominal strain in the x-axis on the stretching sheet, which was measured in the transmitted images. In this cell (referred to as "cell 1"), which had a triangle-like shape, the stress fibers were mainly developed to link between upper and left apexes. In addition, several thick stress fibers were linked between the right apex and other apexes. Figure 3(a) shows the initial state of the cell before stretching. As shown in Figures 3(b)-(f), a uniaxial stretch with magnitude $\mathcal{E}_{s}=10.3 \%$ was applied, and time-lapse images during stretch application were obtained successfully. Figure 4 shows overlaid illustrations of the cell outline in the initial and maximally stretched states on the transmitted image. In Figure 4, the initial state is plotted with a black line and the maximally stretched state is indicated with a red line. Rectangles with vertexes numbered 1 - 4 show deformation of the stretching sheet, which were used to estimate the displacement distribution in cell 1. In this study, we observed three cells and evaluated the deformation behaviors of their stress fibers. Images of initial and maximally 


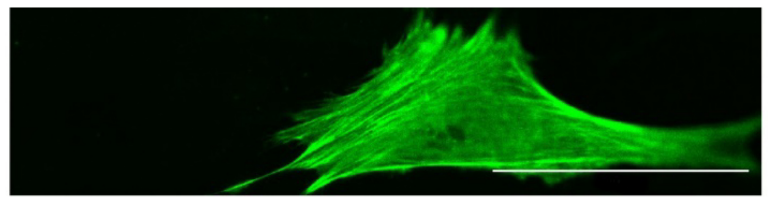

(a)

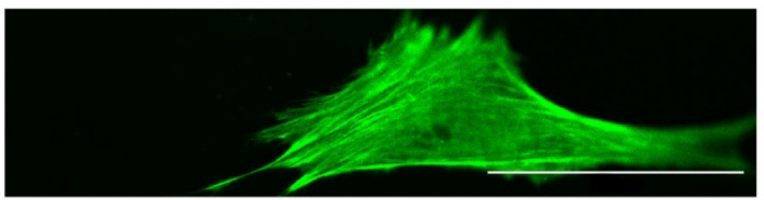

(c)

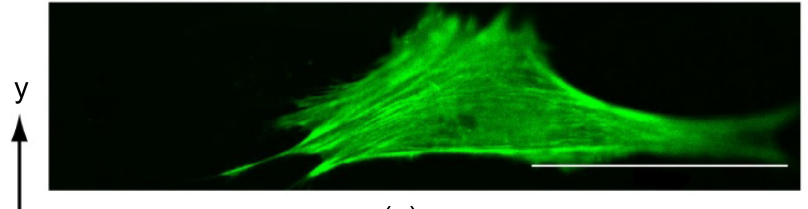

(e)

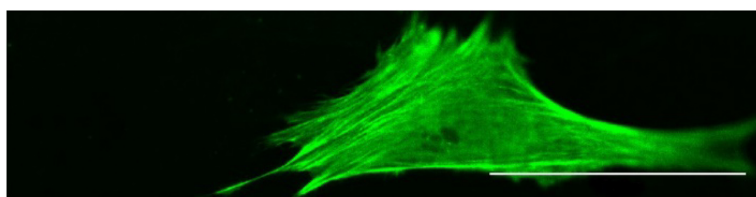

(b)

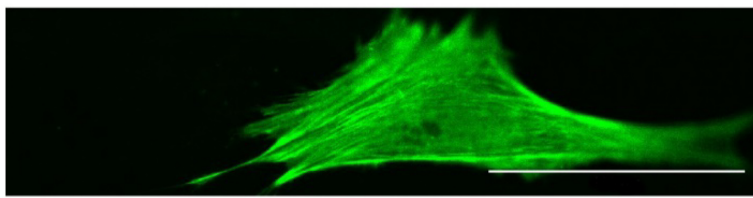

(d)

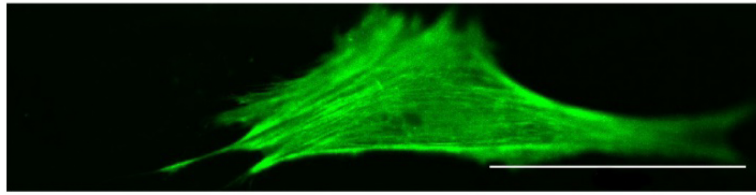

(f)

Figure 3. Time-lapse fluorescence images of Lifeact-tagGFP2 in a stretched cell with $10 \%$ strain. These images are shown with pseudocolor to improve visibility of LUT (green). Uniaxial stretch was applied along the X-axis. Nominal strain $\left(\varepsilon_{s}\right)$ on the stretching sheet was measured in transmitted images obtained simultaneously with fluorescence images. This cell is referred to as "cell 1". Scale bar $=100 \mu \mathrm{m}$. (a) $\varepsilon_{s}=0 \%$; (b) $\varepsilon_{s}=2.17 \%$; (c) $\varepsilon_{s}=4.35 \%$; (d) $\varepsilon_{s}=5.98 \%$; (e) $\varepsilon_{s}=7.88 \%$; (f) $\varepsilon_{s}=10.30 \%$.

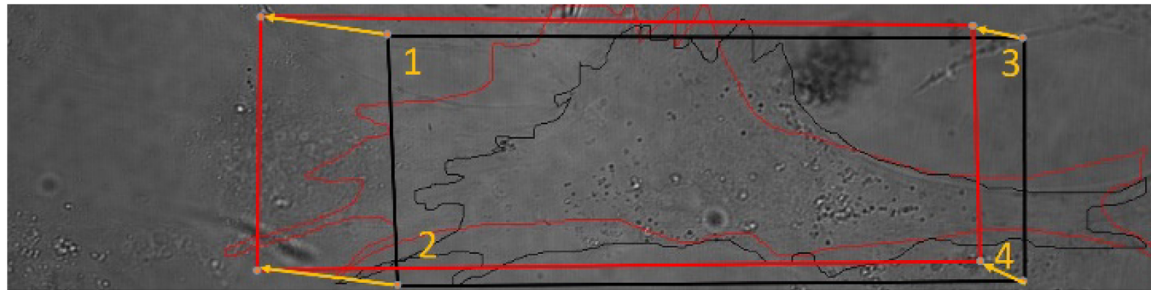

Figure 4. Overlaid cell outline in the initial state (black line) and maximally stretched state (red line) of cell 1 . Traces of representative enclosing spot marks on the stretching sheet used to estimate displacement distribution in cell 1 are shown.

stretched states of other cells are shown in Figure 5(a) Figure 5(b) and Figure 6(a), Figure 6(a). Overlaid illustrations of the cell outline and enclosing rectangles are shown in Figure 5(c) and Figure 6(c).

\subsection{Actual Deformation of Stress Fibers under Uniaxial Stretch}

Using the obtained time-lapse fluorescence images, we performed manual measurement of the fiber angle against the stretch direction and axial strain along the fiber in three to five representative actin stress fibers of cells 1, 2, and 3. Figure 7 shows the configuration of the selected representative actin stress fibers in cell 1 . In the figure, blue lines indicate the traces of measured actin stress fibers. Table 1 shows the measured values of fiber angle $\theta$ and the change in the angle between initial and stretched states $(\varepsilon \theta)$ in cell 1 . Because of uniaxial stretch along the in $\mathrm{x}$-axis and Poisson's effect compression in the $\mathrm{y}$-axis, each stress fiber changed their angle in response to stretch application. Table 2 shows the 


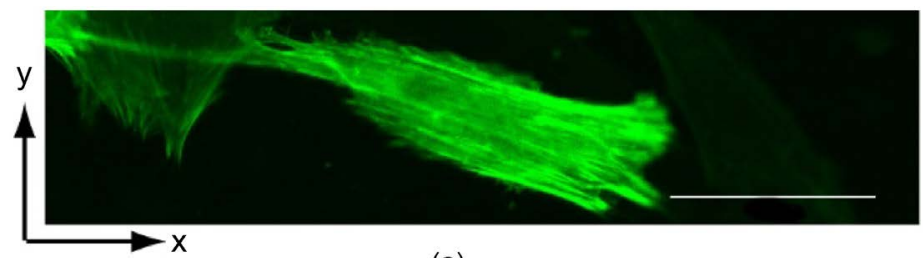

(a)

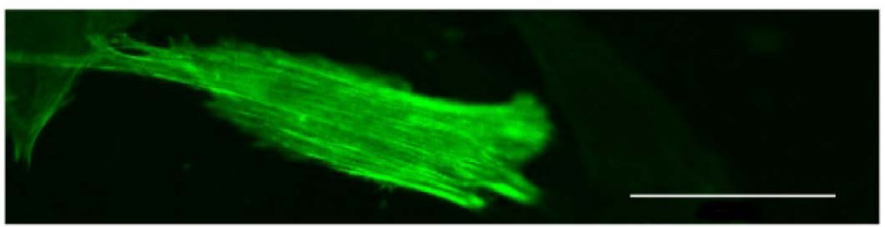

(b)

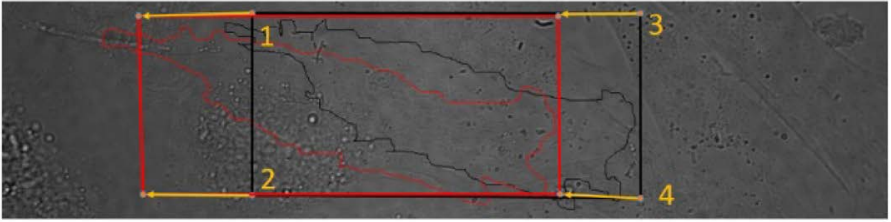

(c)

Figure 5. Fluorescence images of actin stress fibers, overlaid cell outline, and traces of representative enclosing spot markers in the initial and stretched states of "cell 2". $\varepsilon_{s}$ indicates nominal strain on the stretching sheet. Scale bar $=50 \mu \mathrm{m}$. (a) $\mathcal{\varepsilon}_{s}=0 \%$; (b) $\mathcal{\varepsilon}_{s}=6.29 \%$; (c) Cell outline and enclosing rectangle.

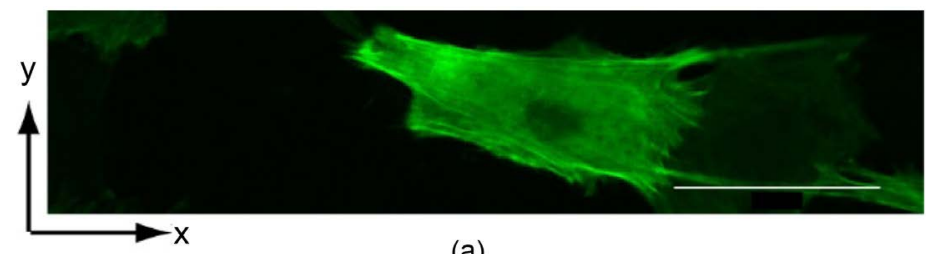

(a)

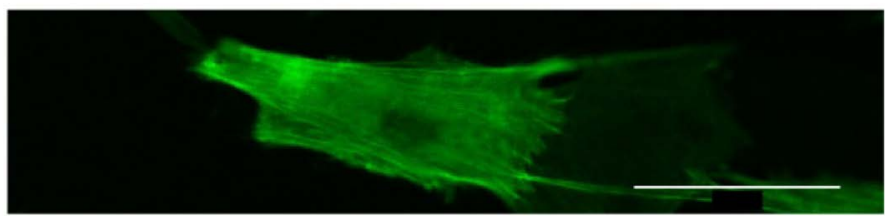

(b)

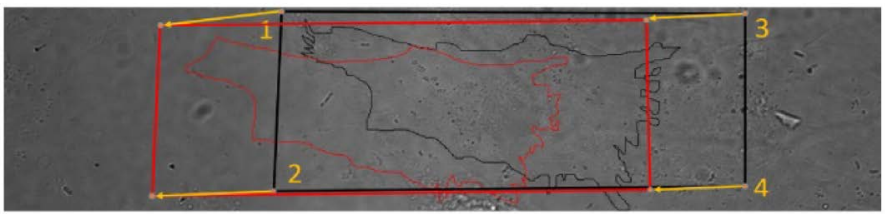

(c)

Figure 6. Fluorescence images of actin stress fibers, overlaid cell outline, and traces of representative enclosing spot markers in the initial and stretched states of "cell 3". $\varepsilon_{s}$ indicates nominal strain on the stretching sheet. Scale bar $=50 \mu \mathrm{m}$. (a) $\varepsilon_{s}=0 \%$; (b) $\varepsilon_{s}=6.16 \%$; (c) Cell outline and enclosing rectangle. 


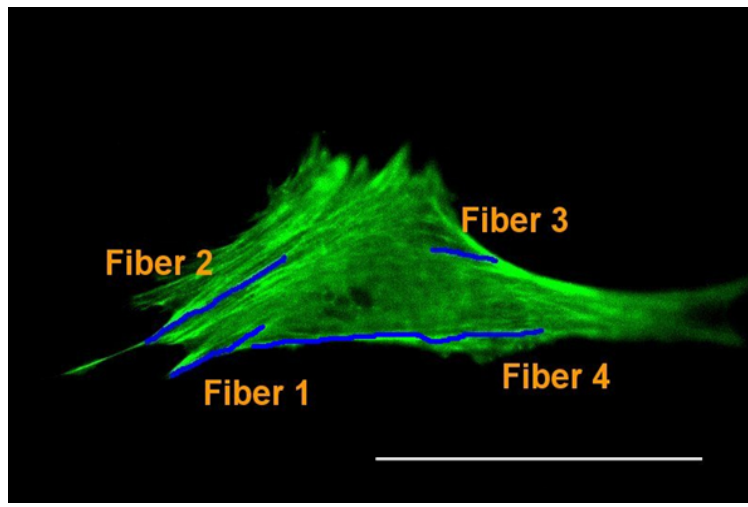

Figure 7. Configurations of manually measured actin stress fibers in cell 1. Blue lines indicate traces of the measured actin stress fibers. Axial strain and the angle of each fiber were evaluated. Scale bar $=100 \mu \mathrm{m}$.

Table 1. Change in the angle of stress fibers and comparison between measured and estimated $\delta \theta$ in "cell 1". The angle is between the fiber axis and stretching direction. $\theta_{1}$ and $\theta_{2}$ are the angles in the initial and stretched states, respectively. Unit $=$ degree.

\begin{tabular}{cccccc}
\hline & $\theta_{1}$ & $\theta_{2}$ & $\delta \theta$ & Estimated $\delta \theta$ & Deviation in $\delta \theta$ \\
\hline Fiber 1 & 28.5 & 24.3 & -4.2 & -3.6 & -0.6 \\
Fiber 2 & 31.5 & 26.0 & -5.5 & -2.7 & -2.8 \\
Fiber 3 & -8.8 & -6.4 & 2.3 & 1.2 & 1.1 \\
Fiber 4 & 2.6 & 1.5 & -1.1 & -2.1 & 1.0 \\
\hline
\end{tabular}

Table 2. Change in the stress fiber length and axial strain, and comparison between measured and estimated $\varepsilon_{F}$ in "cell 1". $L_{1}$ and $L_{2}$ are the lengths in the initial and stretched states, respectively. $\mathcal{E}_{F}$ is the axial fiber strain. Units: $\mu \mathrm{m}$ for length, $\%$ for strain.

\begin{tabular}{cccccc}
\hline & $L_{1}$ & $L_{2}$ & $\varepsilon_{F}$ & Estimated $\varepsilon_{F}$ & Deviation in $\varepsilon_{F}$ \\
\hline Fiber 1 & 34.2 & 36.2 & $5.6 \%$ & $8.4 \%$ & $-2.7 \%$ \\
Fiber 2 & 57.1 & 59.2 & $3.7 \%$ & $8.8 \%$ & $-5.1 \%$ \\
Fiber 3 & 18.7 & 20.3 & $8.7 \%$ & $9.8 \%$ & $-1.1 \%$ \\
Fiber 4 & 83.5 & 89.4 & $7.2 \%$ & $10.3 \%$ & $-3.1 \%$ \\
\hline
\end{tabular}

measured values of axial strain in stress fibers. According to the initial angle of each fiber, the magnitude of axial strain differed between fibers 1 - 4. In fibers 3 and 4 , which were parallel to the stretching direction, the axial strain magnitude was larger than that of fibers 1 and 2 that were tilted against the stretching direction. All fibers showed a smaller strain magnitude compared with the nom nominal strain measured on the stretching sheet. Figure 8 and Figure 9 show the configuration of selected representative actin stress fibers in cells 2 and 3, respectively. In the figures, blue lines indicate the traces of measured actin stress fibers. Table 3 and Table 4 show measured value of the fiber angle and the axial strain magnitude in cell 2. And Table 5 and Table 6 show those of in cell 3. In cell 2, most stress fibers were aligned in the same direction. Therefore, the 


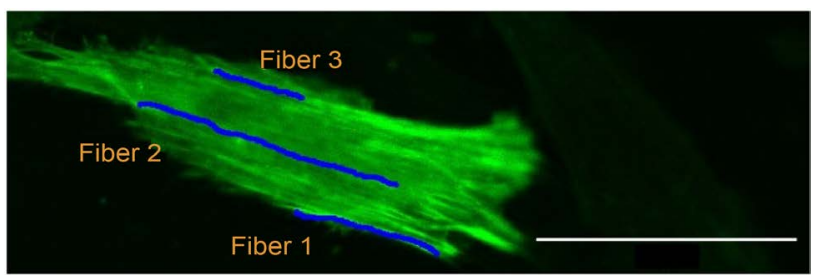

Figure 8. Configurations of manually measured actin stress fibers in cell 2. Blue lines indicate traces of the measured actin stress fibers. Axial strain and the angle of each fiber were evaluated. Scale bar $=50 \mu \mathrm{m}$.

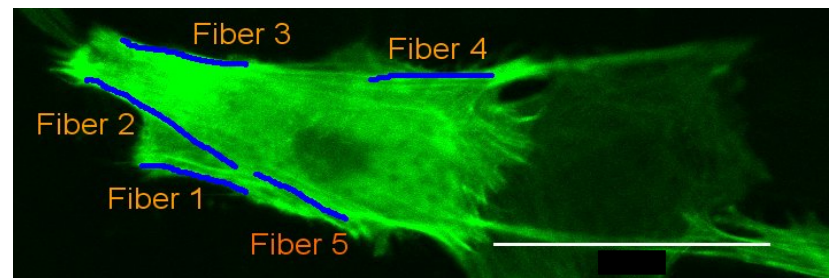

Figure 9. Configurations of manually measured actin stress fibers in cell 3. Blue lines indicate traces of the measured actin stress fibers. Axial strain and the angle of each fiber were evaluated. Scale bar $=50 \mu \mathrm{m}$.

Table 3. Change in the stress fiber angle and comparison between measured and estimated $\delta \theta$ in cell 2". The angle is between the fiber axis and stretching direction. $\theta_{1}$ and $\theta_{2}$ are the angles in initial and stretched states, respectively. Unit = degree.

\begin{tabular}{cccccc}
\hline & $\theta_{1}$ & $\theta_{2}$ & $\delta \theta$ & Estimated $\delta \theta$ & Deviation in $\delta \theta$ \\
\hline Fiber 1 & -17.6 & -15.7 & 1.9 & 2.1 & -0.2 \\
Fiber 2 & -19.3 & -17.2 & 2.1 & 2.0 & 0.1 \\
Fiber 3 & -18.4 & -15.4 & 3.0 & 2.3 & 0.7 \\
\hline
\end{tabular}

Table 4. Change in the stress fiber length and axial strain, and comparison between measured $\varepsilon_{F}$ and estimated $\varepsilon_{F}$ in cell 2. $L_{1}$ and $L_{2}$ are the lengths in initial and stretched states, respectively. $\varepsilon_{F}$ is the axial fiber strain. Units: $\mu \mathrm{m}$ for length; \% for strain.

\begin{tabular}{cccccc}
\hline & $L_{1}$ & $L_{2}$ & $\mathcal{\varepsilon}_{F}$ & Estimated $\mathcal{\varepsilon}_{F}$ & Deviation in $\mathcal{\varepsilon}_{F}$ \\
\hline Fiber 1 & 136.7 & 142.6 & $4.3 \%$ & $6.2 \%$ & $-1.9 \%$ \\
Fiber 2 & 241.0 & 253.7 & $5.3 \%$ & $6.2 \%$ & $-0.9 \%$ \\
Fiber 3 & 80.7 & 84.3 & $4.6 \%$ & $5.3 \%$ & $-0.7 \%$ \\
\hline
\end{tabular}

change in fiber angle $(\varepsilon \theta)$ and axial strain $\left(\varepsilon_{F}\right)$ in each fiber showed a similar trend. Conversely, in cell 3 , fibers 1 and 5 showed a relatively large change in fiber angle $(\varepsilon \theta)$, and fiber 3 showed a relatively large magnitude of axial strain $\left(\varepsilon_{F}\right)$ compared with the other fibers.

\subsection{Comparison between Measured Stress Fiber Deformation and Estimated Deformation of Fibers Calculated from the Surrounding Stretch Sheet Deformation}

In Table 1, Table 3 and Table 5, we compared the change in the fiber angle 
Table 5. Change in the stress fiber angle and comparison between measured and estimated $\delta \theta$ in cell 3 . The angle is between the fiber axis and stretching direction. $\theta_{1}$ and $\theta_{2}$ are the angles in the initial and stretched states, respectively. Unit $=$ degree.

\begin{tabular}{cccccc}
\hline & $\theta_{1}$ & $\theta_{2}$ & $\delta \theta$ & Estimated $\delta \theta$ & Deviation in $\delta \theta$ \\
\hline Fiber 1 & -14.9 & -11.3 & 3.6 & 0.7 & 2.9 \\
Fiber 2 & -30.7 & -28.9 & 1.8 & 2.1 & -0.3 \\
Fiber 3 & -10.9 & -9.8 & 1.1 & 0.5 & 0.6 \\
Fiber 4 & 0.5 & 1.8 & 1.3 & 0.1 & 1.2 \\
Fiber 5 & -28.9 & -26.3 & 2.6 & 2.0 & 0.6 \\
\hline
\end{tabular}

Table 6. Change in the stress fiber length and axial strain, and comparison between measured and estimated $\varepsilon_{F}$ in cell 3. $L_{1}$ and $L_{2}$ are the lengths in the initial and stretched states, respectively. $\varepsilon_{F}$ is axial fiber strain. Units: $\mu \mathrm{m}$ for length; \% for strain.

\begin{tabular}{cccccc}
\hline & $L_{1}$ & $L_{2}$ & $\mathcal{\varepsilon}_{F}$ & Estimated $\mathcal{\varepsilon}_{F}$ & Deviation in $\mathcal{\varepsilon}_{F}$ \\
\hline Fiber 1 & 94.6 & 97.5 & $3.1 \%$ & $6.0 \%$ & $-2.9 \%$ \\
Fiber 2 & 129.1 & 134.7 & $4.3 \%$ & $4.9 \%$ & $-0.6 \%$ \\
Fiber 3 & 105.9 & 111.6 & $5.3 \%$ & $5.4 \%$ & $-0.1 \%$ \\
Fiber 4 & 126.0 & 131.1 & $4.0 \%$ & $5.4 \%$ & $-1.4 \%$ \\
Fiber 5 & 86.8 & 90.3 & $4.0 \%$ & $5.8 \%$ & $-1.8 \%$ \\
\hline
\end{tabular}

between measured and estimated values (deviation in $\varepsilon \theta$ ) in cells 1,2 , and 3 , respectively. In Table 2, Table 4 and Table 6, we compared the axial strain in stress fibers (deviation in $\varepsilon_{F}$ ). As shown in Table 1 and Table 2, there was a relatively large deviation between measured and estimated values in fiber 2 of cell 1 . Conversely, as shown in Table 3 and Table 4, the measured and estimated values were in accordance with both $\varepsilon \theta$ and $\varepsilon_{F}$ in cell 2 . In cell 3, only fiber 1 showed a large deviation in both $\varepsilon \theta$ and $\varepsilon_{F}$ (Table 5 and Table 6).

\section{Discussion}

In the present study, we successfully conducted in situ observation of actin stress fiber dynamics under uniaxial stretch application. In some cells with nonhomogeneous stress fiber structure, the distribution of axial strain and change in fiber angle were not uniform, and there were relatively larger deviations in the measured axial strain and change in fiber angle compared with their estimated values that were calculated according to stretching sheet deformation. Conversely, in the cell with a homogeneous stress fiber structure, such as alignment in one direction, axial strain and the fiber angle change were relatively uniform and matched with the estimated values. These results suggest that mechanical stimulation, such as stretching of the extracellular matrix, is transmitted into a cell non-uniformly under the influence of a complex actin stress fiber structure. Furthermore, in the region around the cell nucleus, there was a more complicated local strain distribution compared with other regions. For example, fiber 2 in cell 1, which ran from the edge 
of the cell to the cell nucleus, had a relatively large deviation of axial strain compared with other regions. We speculate that the cell nucleus with a stiffer mechanical property [9] affects such a nonhomogeneous strain distribution in stress fibers. Further understanding of the transmission characteristics of mechanical force and deformation via actin stress fibers will help us to clarify the mechanosensing mechanism of cells.

Guolla et al. also have reported the strain dynamics in actin stress fibers in response to applied external force [10]. They conducted simultaneous observation of atomic force microscopy (AFM) and fluorescent microscopy, and evaluated the precise strain dynamics in actin stress fibers. In their experimental setup, external force was applied to cell nucleus by using the AFM probe. Although their findings are very useful to understand force transmitting characteristic of actin stress fiber network in the cell, the external force was applied by pushing the cell nucleus artificially. Therefore it is not very appropriate to discuss the force and deformation transmitting characteristics of biological mechanical stimulus such as stretching of extracellular matrix on which cells adhere. An advantage of our study is that we succeeded in conducting the in situ strain measurement of actin stress fibers in response to biological mechanical stimulus.

To obtain time-lapse images with high spatial resolution, the image scanning rate was set to very low. In addition, the stretch strain rate was slow, like quasistatic stretching, to suppress distortion of the image. It is widely known that cellular components, such as actin fibers, the cell membrane and nucleus, have viscoelastic properties [11] [12] [13]. Therefore, the effect of the stretch strain rate has to be taken into account. If we had applied stretching deformation at a higher strain rate, the results of the stress fiber dynamics might have been different.

There are several cell-stretching devices that aim to conduct in situ observation of cells under stretch application [14]. A noticeable advantage of our stretching MEMS device is that it can hold a target cell in not only the horizontal plane but also the depth direction (focus axis) during stretch application. Focus drift during stretch application yields a considerable influence on the accuracy of data analysis. The amount of focus drift during stretch application was estimated to be approximately 1 to 2 microns by refocusing to the target cell after observation (data not shown). Although this focus drift of several microns is sufficiently small to conduct manual measurement of the fiber which has noticeable branches or nodes for clear tracing marker, it is not sufficiently small to perform digital image correlation (DIC) method-based strain measurement. We attempted to measure the strain distribution in cells using by using DIC based method, but we could not confirm the accuracy of the obtained strain distribution because even a focus drift of several microns causes a considerable change in the pixel pattern and reduces the accuracy of DIC measurements. To conduct further investigation of the strain distribution in cells under stretch application, the observational setup has to extend to a four dimensional time-course measurement including the $\mathrm{z}$-axis (focus drive) in future studies. 


\section{Acknowledgements}

Authors would like to thank Professor Kazuki Horikawa for his technical support and generous advice. This study was technically supported by the Support Center for Advanced Medical Sciences, Institute of Health Biosciences, Tokushima University Graduate School. In addition, this study was partly supported by JSPS KAKENHI Grant Number 26350503.

\section{References}

[1] Lauffenburger, D.A. and Horwitz, A.F. (1996) Cell Migration: A Physically Integrated Molecular Process. Cell, 84, 359-369. https://doi.org/10.1016/S0092-8674(00)81280-5

[2] Goldyn, A.M., Rioja, B.A., Spatz, J.P., Ballestrem, C. and Kemkemer, R. (2009) ForceInduced Cell Polarization Is Linked to RhoA-Driven Microtubule-Independent FocalAdhesion Sliding. Journal of Cell Science, 122, 3644-3651. https://doi.org/10.1242/jcs.054866

[3] Shafrir, Y. and Forgacs, G. (2002) Mechanotransduction through the Cytoskeleton. American Journal of Physiology, Cell Physiology, 282, C479-C486. https://doi.org/10.1152/ajpcell.00394.2001

[4] Toma, C.D., Ashkar, S., Gray, M.L., Schaffer, J.L. and Gerstenfeld, L.C. (1997) Signal Transduction of Mechanical Stimuli Is Dependent on Microfilament Integrity: Identification of Osteopontin as a Mechanically Induced Gene in Osteoblasts. Jour nal of Bone Mineral Research, 12, 1626-1636. https://doi.org/10.1359/jbmr.1997.12.10.1626

[5] Chen, N.X., Ryder, K.D., Pavalko, F.M., Turner, C.H., Burr, D.B., Qiu, J. and Duncan, R.L. (2000) $\mathrm{Ca}^{2+}$ Regulates Fluid Shear-Induced Cytoskeletal Reorganization and Gene Expression in Osteoblasts. American Journal of Physiology. Cell Physiology, 278, C989-C997.

[6] Greiner, A.M., Chen, H., Spatz, J.P., Ballestrem, C. and Kemkemer, R. (2013) Cyclic Tensile Strain Controls Cell Shape and Directs Actin Stress Fiber Formation and Focal Adhesion Alignment in Spreading Cells. PLoS ONE, 8, e77328. https://doi.org/10.1371/journal.pone.0077328

[7] Gerstmair, A., Fois, G., Innerbichler, S., Dietl, P. and Felder, E. (2009) A Device for Simultaneous Live Cell Imaging during Uniaxial Mechanical Strain or Compression. Journal of Applied Physiology, 107, 613-620. https://doi.org/10.1152/japplphysiol.00012.2009

[8] Sato, K., Kamada, S. and Minami, K. (2010) Development of Microstretching Device to Evaluate Cell Membrane Strain Field around Sensing Point of Mechanical Stimuli. International Journal of Mechanical Sciences, 52, 251-256. https://doi.org/10.1016/j.ijmecsci.2009.09.021

[9] Caille, N., Thoumine, O., Tardy, Y. and Meister, J.-J. (2002) Contribution of the Nucleus to the Mechanical Properties of Endothelial Cells. Journal of Biomechanics, 35, 177-187. https://doi.org/10.1016/S0021-9290(01)00201-9

[10] Guolla, L., Bertrand, M., Haase, K. and Pelling, A.E. (2011) Force Transduction and Strain Dynamics in Actin Stress Fibers in Response to Nanonewton Forces. Journal of Cell Science, 125, 603-613. https://doi.org/10.1242/jcs.088302

[11] Guilak, F., Tedrow, J.R. and Burgkart, R. (2000) Viscoelastic Properties of the Cell Nucleus. Biochemical and Biophysical Research Communications, 269, 781-786. https://doi.org/10.1006/bbrc.2000.2360 
[12] Kumar, S., Maxwell, I.Z., Heisterkamp, A., Polte, T.R., Lele, T.P., Salanga, M., Mazur, E. and Ingber, D.E. (2006) Viscoelastic Retraction of Single Living Stress Fibers and Its Impact on Cell Shape, Cytoskeleton Organization, and Extracellular Matrix Mechanics. Biophysical Journal, 90, 3762-3773.

https://doi.org/10.1529/biophysj.105.071506

[13] Lu, L., Oswald, S.J., Ngu, H. and Yin, F.C.-P. (2008) Mechanical Properties of Actin Stress Fibers in Living Cells. Biophysical Journal, 95, 6060-6071.

https://doi.org/10.1529/biophysj.108.133462

[14] Kamble, H., Barton, M.J., Jun, M., Park, S. and Nguen, N. (2016) Cell Stretching Devices as Research Tools: Engineering and Biological Considerations. Lab on a Chip, 16, 3193-3203. https://doi.org/10.1039/C6LC00607H 